\title{
Epidemiology of resistance and phenotypic characterization of carbapenem resistance mechanisms in Klebsiella pneumoniae isolates at Sahloul University Hospital-Sousse, Tunisia
}

\author{
Aziza Messaoudi ${ }^{1}$, Wejdène Mansour ${ }^{2,3}$, Nedia Jaidane ${ }^{1}$, \\ Chrifa Chaouch $^{1,4}$, Noureddine Boujaâfar ${ }^{4}$, Olfa Bouallègue ${ }^{3}$
}

1. Research Unit: Emergent Bacterial Resistance and Safety of Care (UR12SP37), Laboratory of Microbiology, University Hospital Sahloul, Sousse, Tunisia.

2. Laboratory of Metabolic Biophysics and Applied Pharmacology (LR12ES02), Department of Biophysics,

Faculty of Medicine Ibn El Jazzar of Sousse, University of Sousse, Sousse, Tunisia

3. Faculty of Medicine Ibn Al Jazzar, Sousse, University of Sousse, Tunisia.

4. Faculty of Pharmacy, Monastir, University of Monastir, Monastir, Tunisia

\begin{abstract}
Objective: To assess the prevalence of ESBL producing and carbapenem resistant Klebsiella pneumoniae isolated from in-come and out-come patients at Sahloul-university hospital.

Methods: A retrospective study over a 3 years period (January 2012 and December 2014) focused on 2160 strains of Klebsiella pneumoniae. Statistical analysis was carried out using SPSS program. ESBL detection was performed using a double disc diffusion method and carbapenemase detection was realized by Rosco-Disk kit.

Results: A total of 2160 Klebsiella pneumoniae strains were isolated during the period of the study, 26.2\% ( $\mathrm{n}=566$ ) were ESBL-producers and $15.8 \%(n=342)$ showed resistance to carbapenem. The wards most affected by these strains were basically urology and intensive care units. Eighty four percent of studied strains (203/241) were resistant to temocillin, which correlate with the production of a class D (OXA-48-like) carbapenemase and 7\% (17/241) showed sensitivity to EDTA and dipicolinic acid, which indicate the production of metallo-enzyme. The rate of resistance to colistin remains low.

Conclusion: Resistance of Enterobacteriaceae, including K. pneumoniae, to third generation cephalosporins ( $\left.3^{\text {rd }} \mathrm{GC}\right)$ and carbapenem through the mechanism of ESBL and carbapenemases production is becoming increasingly worrying. This suggests a more rational use of antibiotics, as well as the rigorous application of hygiene measurement.
\end{abstract}

Keywords: Klebsiella pneumoniae, epidemiology, ESBL, carbapenemase, phenotypic screening.

DOI: https://dx.doi.org/10.4314/ahs.v19i2.24

Cite as: Messaoudi A, Mansour W, Jaidane N, Chaouch C, Boujaâfar N, Bouallègue O. Epidemiology of resistance and phenotypic characterization of carbapenem resistance mechanisms in Klebsiella pneumoniae isolates at Sabloul University hospital-Sousse, Tunisia. Afri Health Sci.2019;19(2): 2008-2020. bttps://dx.doi.org/10.4314/abs.v19i2.24

\section{Introduction}

Hospital infections have become a growing healthcare challenge in recent decades and serious concerns have

\begin{tabular}{|l|}
\hline Corresponding author: \\
Wejdene Mansour, \\
Laboratory of Metabolic Biophysics \\
and Applied Pharmacology (LR12ES02), \\
Department of Biophysics, \\
Faculty of Medicine Ibn El Jazzar of Sousse, \\
University of Sousse, Sousse, Tunisia. \\
Phone: +216-97-22-66-52; \\
Fax: 216-73-68-21-90. \\
Email: wejdene.mansour@gmail.com \\
\hline
\end{tabular}

been expressed over the rise in antimicrobial resistance among pathogens causing hospital-acquired infections. The progression of bacterial resistances to antibiotics confronts clinicians with infections that are difficult to treat and poses a public health problem.

In view of emergence of ESBL and AmpC-producing bacteria, carbapenems are the $\beta$-lactam group of drugs that are often used as antibiotics of last resort and have shown their stability for treating infection due to multidrug-resistant bacteria ${ }^{1}$. As consequence of over-use of this treatment, the situation has changed with the emergence of carbapenem- resistant bacteria both in fermenters Gram-negative bacilli (Enterobacteriaceae) and non-fer- 
menters bacteria (Acinetobacter baumannii and Pseudomonas aeruginosa $)^{2}$. Gram-negative bacteria play a significant role and have the strongest impact on the development and the emergence of antimicrobial resistance.

Resistance to carbapenems is mediated by two principal mechanisms: (i) production of ESBL and/or AmpC enzymes with non-significant carbapenemase activity combined with loss of porin or up-regulation of efflux pump (ii) secretion of carbapenem-hydrolyzing $\beta$-lactamases.

The risk of spreading of Enterobacteriaceae producing carbapenemases is a major public health issue, as these enzymes restrict treatment options and are often associated with other mechanisms, conferring multi-resistance strains ${ }^{3}$. Invasive infections with these organisms have been associated with high rates of morbidity and mortality ${ }^{4}$. Despite the recommendations concerning the rational use of antibiotics and the strengthening of hygiene procedures to limit the emergence of multidrug resistant bacteria, the number of epidemics due to carbapenemase-producing bacteria has been increasing in several countries throughout the world. Tunisia was not spared were carbapenemases producing Enterobacteriaceae has increased in a spectacular way. The first report of carbapenemases bacterial production in Tunisia was in $2010^{5}$. The most described carbapenemases can belong to clavulanic-acid inhibited $\beta$-lactamases (KPC), metallo- $\beta$-lactamases $(\mathrm{NDM})$ or expended spectrum oxacillinases (OXA-48).

The aim of this study was to describe the general state of the evolution of resistance in Enterobacteriaceae and especially in Klebsiella pneumoniae isolated in the laboratory of microbiology in Sahloul hospital and to phenotypically elucidate the mechanisms responsible for resistance to the molecules of carbapenems.

\section{Global methodology of the undertaken survey}

A retrospective investigation was carried out over a period of three years from $01 / 01 / 2012$ to $31 / 12 / 2014$ in the laboratory of microbiology in Sahloul hospital, a 629 beds university hospital with surgical vocation. The descriptive part of this study concern epidemiology of resistance to antibiotics in Enterobacteriaceae and statistical analysis was carried out using the SPSS software (version 20) and data from the laboratory. Phenotypic detection of the mechanisms responsible for carbapenem resistance was carried out on Klebsiella pneumoniae strains isolated from the various samples taken from hospitalized patients and patients consulting in the external services of the hospital (emergencies, consultations urology, nephrology, gastrology).

Anti-microbial susceptibility testing was performed by classic method of disk diffusion on Mueller-Hinton agar plates. This test was performed for all isolates. Different classes of antibiotics were tested including $\beta$-lactam and non $\beta$-lactam antibiotic containing disks (Bio-Rad, France). The antibiotics used were: ampicillin, piperacillin, ticarcillin, ampicillin-clavulanic acid, piperacillin-tazobactam, ticarcillin-clavulanic acid, cephalotin, cefuroxime, cefepime, cefoxitin, cefotaxime, ceftazidime, ertapenem, imipenem, aztreonam, gentamicin, amikacin, kanamycin, netilmicin, tobramycin, tetracyclin, ciprofloxacin, levofloxacin, ofloxacin, nalidixic acid, colistin, sulfonamides, trimethoprim and chloramphenicol. Results were interpreted according to the guidelines of the European committee on Anti-microbial Susceptibility testing (EUCAST 2015; www.eucast.org).

To detect ESBL producing strains, double disc synergy test was used: the picture of synergy between the disc of third generation cephalosporin antibiotic (ticarcillin) and other containing clavulanic acid (ampicillin-clavulanic acid) confirm the presence of ESBL enzyme. Phenotypic detection of carbapenemases producing strains was carried out for ertapenem resistant strains using ROSCO KPC/MBL and OXA-48 Confirm Kit (ROSCO Diagnostica, Taastrup, Denmark) as a perform phenotypic test to elucidate the presence and the class of carbapenemases. Principle of this test was to compare diameter of resistance of meropenem alone and in combination with different inhibitors: dipicolinic acid against metallo- $\beta$-lactamases, aminophenylboronic acid against Ambler class A $\beta$-lactamase and cloxacillin against AmpC cephalosporinase. Temocillin was used for presumptive detection of Ambler class D oxacillinases enzymes producers (table 1). 
Table 1: Interpretation of disc ROSCO results

\begin{tabular}{|l|l|l|l|l|}
\hline & MRP+Cloxacilline & MRP+PBA & MRP+Dip.acid & Temocillin \\
\hline Class A carbapenemases & - & $+(\geq 5 \mathrm{~mm})$ & - & - \\
\hline Class B carbapenemases & - & - & $+(\geq 5 \mathrm{~mm})$ & $+/-$ \\
\hline Class D carbapenemases & - & - & - & + \\
\hline AmpC+porin loss & $+(\geq 5 \mathrm{~mm})$ & $+/-$ & - & - \\
\hline
\end{tabular}

MRP: Meropenem; PBA: Aminophenylboronic acid; Dip.acid: Dipicolinic acid; AmpC: Cephalosporinase

\section{Results and discussion}

\section{A- General features}

Enterobacteriaceae is a large family that includes several species which are the most commonly isolated between human pathogens in a bacteriological laboratory, in both community and hospital settings. They are responsible of many diseases such as urinary, respiratory and abdominal tract infections and septicemia ${ }^{6,7}$. Escherichia and Proteus predominate in the intestinal commensal flora. They sometimes act asopportunistic pathogens. The Klebsiella, Enterobacter, Citrobacter, Serratia, Morganella and Providencia genera found in wastewater, soils and in small quantities in the gastrointestinal tract can cause serious infections due to their resistance to antibiotics, particularly in hospitals ${ }^{8}$.
At Sahloul hospital in Sousse, Enterobacteriaceae are also among the most isolated species in microbiological laboratory. These bacteria are mainly responsible for urinary infections. It is known that urinary infections with Enterobacteriaceae are due to the migration of these germs from the digestive tract to the urinary tree. The anatomical reasons explain their greater frequency in the woman but all the causes of stasis (lithiasis, compression, pregnancy) constitute factors favoring the infection.

During the period of study, a total of 9054 strains of Enterobacteriaceae were isolated in the laboratory, Escherichia coli is the most isolated species followed by Klebsiella pneumoniae both at community and nosocomial infections (Table 2).

Table 2: Distribution of Enterobacteriaceae per year of study according to the different species

\begin{tabular}{|c|c|c|c|c|c|c|c|}
\hline \multirow[t]{2}{*}{2012} & \multirow[b]{2}{*}{$\begin{array}{l}\text { Community } \\
\text { strains }\end{array}$} & \multicolumn{2}{|l|}{2013} & \multicolumn{2}{|l|}{2014} & \multirow{2}{*}{ Total } & \\
\hline & & $\begin{array}{l}\text { Nosocomial } \\
\text { strains }\end{array}$ & $\begin{array}{l}\text { Community } \\
\text { strains }\end{array}$ & $\begin{array}{l}\text { Nosocomial } \\
\text { strains }\end{array}$ & $\begin{array}{l}\text { Community } \\
\text { strains }\end{array}$ & & $\begin{array}{l}\text { Nosocomial } \\
\text { strains }\end{array}$ \\
\hline Escherichia coli & 940 & 503 & 1131 & 593 & 1066 & 686 & 4919 \\
\hline $\begin{array}{l}\text { Klebsiella } \\
\text { pneumoniae }\end{array}$ & 370 & 301 & 465 & 325 & 421 & 278 & 2160 \\
\hline Enterobacter cloacae & 82 & 125 & 74 & 123 & 79 & 135 & 618 \\
\hline Morganella morganii & 31 & 28 & 22 & 32 & 18 & 42 & 173 \\
\hline Klebsiella oxytoca & 16 & 43 & 18 & 44 & 20 & 28 & 169 \\
\hline Klebsiella spp & 4 & 6 & 1 & 4 & 1 & 1 & 17 \\
\hline Others species & 71 & 193 & 200 & 204 & 131 & 199 & 998 \\
\hline Total & 1514 & 1199 & 1911 & 1325 & 1736 & 1369 & 9054 \\
\hline
\end{tabular}


B-Klebsiella pneumoniae in Sahloul university hospital-Tunisia

According to the high incrimination of Klebsiella pneumoni$a e$ in nosocomial and community-acquired infections and to the alarming and evolutionary profile of resistance to antibiotics, our study was focused on the description of the epidemiology of Klebsiella pneumoniae strains.

In our hospital, 2160 Klebsiella pneumoniae were selected during three years: $1250 / 2160(43 \%)$ were isolated from patients hospitalized in different wards and 910/2160
$(57 \%)$ were isolated from external consultant patients. Klebsiella pneumoniae is a common pathogen that causes both community-acquired and hospital-acquired infections associated with high morbidity and mortality rates 9 . Post-surgery and intensive care units present the wards where we observe most infections and resistant strains (table 3), followed by nephrology and urology which have also the most important number of Klebsiella pneumoniae isolated strains both in terms of internal and external consultation services (Figure 1,2).

Ward

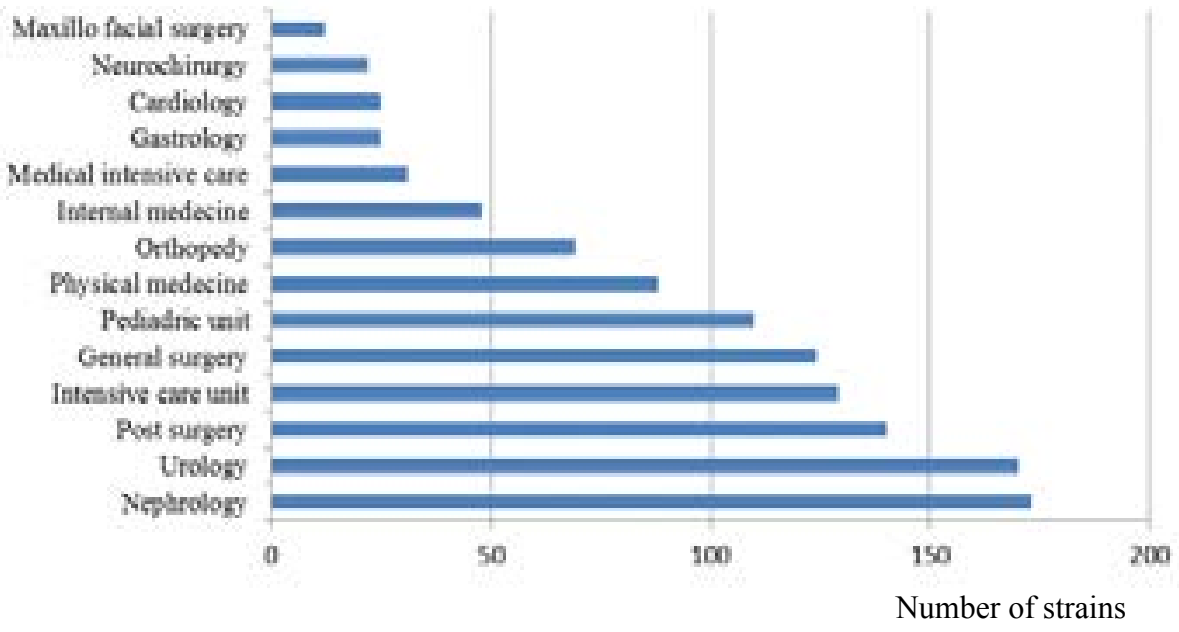

Figure 1: Distribution of Klebsiella pneumoniae in internal wards in the university hospital Sahloul, Sousse Tunisia (2012-2014)

External consultation service

Number of strains

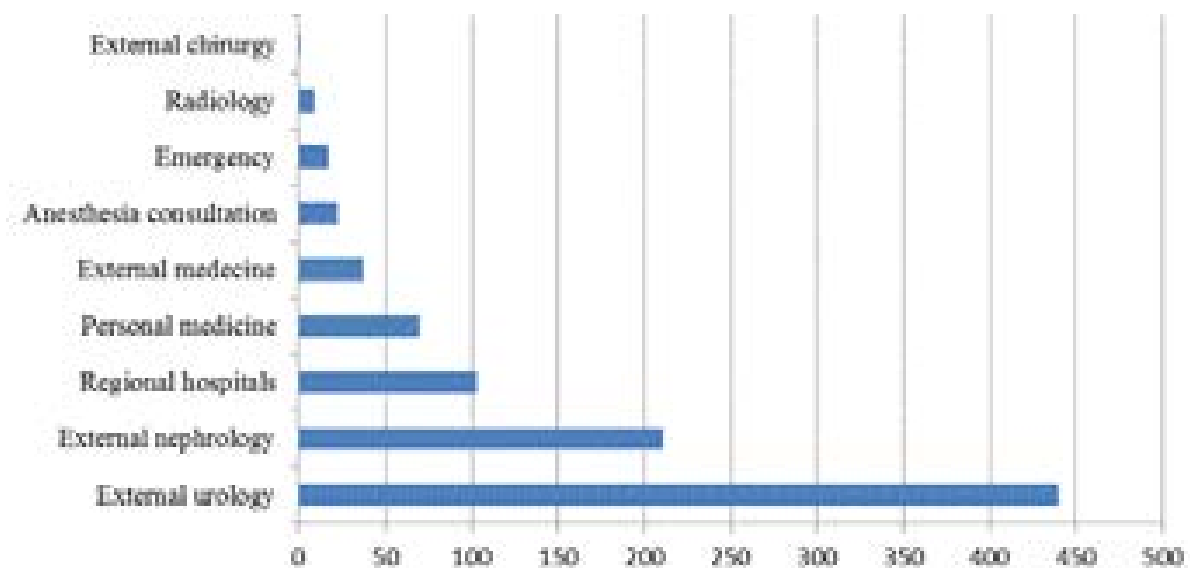

Figure 2: Distribution of Klebsiella pneumoniae in external consultation services

(community-acquired strains) (2012-2014) 
According to the type of specimen, Klebsiella pneumoniae strains are essentially isolated from urine (71\%), especially in women (892/1551 urine samples). Nevertheless, the frequency of isolation in blood remains high $(12 \%)$ (Figure 3). The most frequent infections sites with Klebsiella pneumoniae are the respiratory and urinary tracts. $K$. pneumoniae can cause infections in young and very old patients; however, they are most common in those whose immune system is compromised either through disease (alcoholism, diabetes) or therapy (antimetabolites) 9, 10. C- Antibiotic resistance in Klebsiella pneumoniae strains isolated in the universityhospital Sahloul Tunisia (2012-2014)

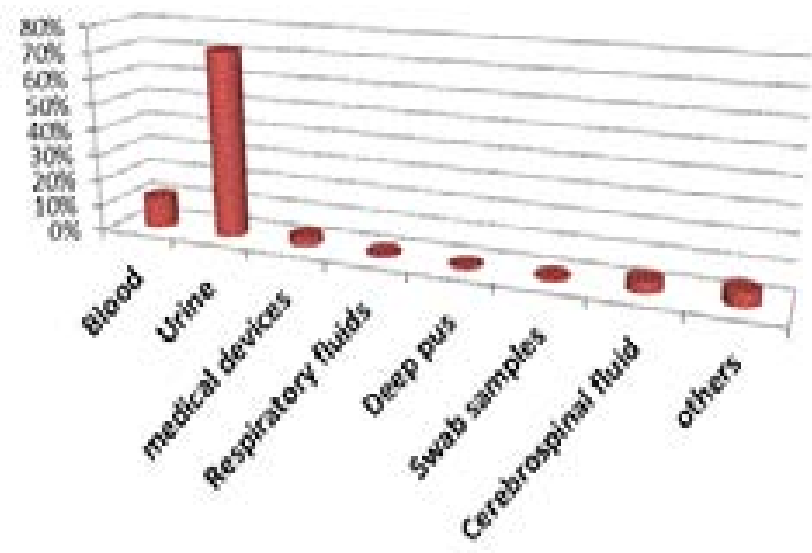

Figure 3: Distribution of Klebsiella pneumoniaeaccording to nature of samples

The advent of antibiotic therapy in the 1940s led to revolution of medical field and to a significant reduction in mortality associated with infectious diseases. Unfortunately, bacterial resistance to traditional antibiotics has rapidly constituted a major health problem on a global scale. The resistance to penicillin was described in the 1950s, to the first-generation cephalosporins in the 1970s and to the third generation cephalosporins in the 1990s. In recent years, the frequency of infections caused by resistant bacteria has increased both in hospitals and in community. Resistance can now be observed in almost all potentially pathogenic bacteria ${ }^{11}$.

In regard to Klebsiella pneumoniae, this species belong to group 2 of Enterobacteriaceae, which include species naturally resistant to amino and carboxypenicillins ${ }^{12}$.
In our study, and based on the antibiogram profiles, $38.6 \%(834 / 2160)$ of studied strains presented the wild phenotype, meaning resistance to amino and carboxypenicillins. This specie is an important source of transferable antibiotic resistance and several hospital outbreaks of infection are caused by multiresistant Klebsiella pneumoniae strains. Third generation cephalosporins such as cefotaxim, ceftriaxone and ceftazidime, are used as a very effective treatment against infections caused by Enterobacteriaceae. Their prescription is generally reserved for severe infections. Similarly, they are used with some success in nosocomial infections. The over use of these molecules, led to the appearance of resistant strains by the production of ESBL enzymes: $26.2 \%(566 / 2160)$ of studied stains correspond to the phenotype of ESBL producers Klebsiella pneumoniae. 
Table 3: distribution of admissions between services during the years (2012-2013 and 2014)

\begin{tabular}{|c|c|c|c|c|c|c|}
\hline Wards & $\begin{array}{l}\text { Total of admission } \\
(2012)\end{array}$ & $\begin{array}{l}\begin{array}{l}\text { Nosocomial } \\
\text { K.pneumoniae } \\
(2012)\end{array} \\
\end{array}$ & $\begin{array}{l}\text { Total of admission } \\
\text { (2013) }\end{array}$ & $\begin{array}{l}\begin{array}{l}\text { Nosocomial } \\
\text { K.pneumoniae } \\
\text { (2013) }\end{array} \\
\end{array}$ & $\begin{array}{l}\text { Total of } \\
\text { admission (2014) }\end{array}$ & $\begin{array}{l}\text { Nosocomial } \\
\text { K.pneumoniae } \\
\text { (2014) }\end{array}$ \\
\hline Nephrology & 1262 & 47 & 1317 & 71 & 1331 & 55 \\
\hline Urology & 3055 & 72 & 2862 & 53 & 2940 & 45 \\
\hline $\begin{array}{l}\text { Intensive care unit and } \\
\text { Post-surgery }\end{array}$ & 1119 & 106 & 985 & 90 & 1110 & 73 \\
\hline General surgery & 3248 & 40 & 3316 & 38 & 3218 & 46 \\
\hline Pediatric unit & 1591 & 30 & 1748 & 39 & 2039 & 41 \\
\hline Physical medicine & 150 & 31 & 226 & 20 & 216 & 37 \\
\hline Orthopedy & 6073 & 15 & 5318 & 34 & 5318 & 20 \\
\hline Internal medecine & 599 & 8 & 588 & 18 & 523 & 22 \\
\hline Medical intensive care & 86 & 9 & 79 & 8 & 85 & 14 \\
\hline Gastrology & 898 & 5 & 894 & 10 & 925 & 10 \\
\hline Cardiology & 2793 & 6 & 2694 & 11 & 2471 & 8 \\
\hline Neuro surgery & 835 & 4 & 916 & 10 & 849 & 8 \\
\hline Maxillo facial surgery & 1712 & 0 & 1586 & 6 & 1589 & 6 \\
\hline Other wards & 8085 & - & 7100 & - & 5814 & - \\
\hline Total of admission & 31506 & - & 29629 & - & 28428 & - \\
\hline
\end{tabular}

In front of the emergence of $\beta$-lactamase and multiresistant strains to several families of antibiotics, carbapenem was used as last-resort molecules for the treatment of infections due to multidrug-resistant bacteria, as consequence, carbapenem resistant strains were emerged. In our study, $15.8 \%(342 / 2160)$ of studied strains cor- respond to the phenotype of carbapenemase producers strains.

Besides ESBL and carbapenemases phenotypes, several other type of resistance can be described in Klebsiella pneumoniae including high-level penicillinase-producing strains, hyper produced cephalosporinases, aminoglycoside and fluoroquinolone-resistant strains (table 4).

Table 4: Distribution of resistance phenotypes during the studied period

\begin{tabular}{|c|c|c|c|c|c|}
\hline & 2012 & 2013 & 2014 & Total \\
\hline & & Number $(\%)$ & Number $(\%)$ & Number (\%) & Number (\%) \\
\hline \multirow{4}{*}{ 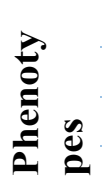 } & Wild-type & $254(37,8 \%)$ & $316(40,0 \%)$ & $264(37,8 \%)$ & $834(38,6 \%)$ \\
\hline & Carbapenemase & $119(17,7 \%)$ & $78(10,0 \%)$ & $145(20,7 \%)$ & $342(15,8 \%)$ \\
\hline & ESBL & $186(27,8 \%)$ & $231(29,2 \%)$ & $149(21,3 \%)$ & $566(26,2 \%)$ \\
\hline & Others & $112(16,7 \%)$ & $165(20,8 \%)$ & $141(20,2 \%)$ & $418(19,3 \%)$ \\
\hline \multicolumn{2}{|l|}{ Total } & $671(100 \%)$ & $790(100 \%)$ & $699(100 \%)$ & $2160(100 \%)$ \\
\hline
\end{tabular}

\section{a-Resistance to cephalosporins}

Cephalosporins are antibiotics belonging to the large family of $\beta$-lactams. These molecules had been developed in response to the increased prevalence of $\beta$-lactamases like TEM-1 and SHV-1 ${ }^{13}$. The molecules which can be used in medicine are semisynthetic derivatives of cephalosporin C; the first was cefalotin, discovered in 1962, belongs to the first-generation cephalosporins14. Other derivatives have been progressively discovered to the current range of cephalosporins. Among the most used molecules in 
therapeutics, we found cefuroxim and cefoxitin belongs to the second-generation cephalosporins, and cefotaxim belongs to third generation cephalosporins. The spectrum of action of successive generations of cephalosporins is different. The first generation has a spectrum mostly oriented towards Gram positive bacteria, the second has an intermediate spectrum and the third generation sees its spectra widen towards Gram negative bacteria.

Klebsiella pneumoniae exhibits high resistance levels to most antibiotics used for treatment, in particular to $3^{\text {rd }}$ GC. In our study, $43.8 \%$ were resistant to cefalotin, $43.8 \%$ to ce- furoxime, $12.1 \%$ to cefoxitin and $41 \%$ to cefotaxim.

The ESBL-producing strains represent $26.2 \%$ of the total of studied Klebsiella pneumoniae. These strains are very well represented in both hospital and community settings (Table 4). The percentage of ESBL-producing strains isolated from hospitalized patients is slightly higher than that of strains from outpatients (Table 5). Initially, ESBL enzymes were restricted to nosocomial strains, they are recognized as major causes of nosocomial acquired infections, but now this is no longer the case, ESBL has emerged in an alarming way in the community and poses a real public health problem ${ }^{15}$.

\section{Table 5: Distribution of ESBL-producing strains between in-come and out-come patients}

\begin{tabular}{lll}
\hline & Number of strains & $\%$ \\
\hline Community strains & 227 & 40,1 \\
\hline Nosocomial strains & 339 & 59,9 \\
\hline Total & 566 & 100,0 \\
\hline
\end{tabular}

The ESBL-producing strains are mostly located in urology and nephrology departments. Intensive care units are also concerned followed by pediatric unit, post-surgery and general surgery department. The emergence of ESBL-producing strains especially in these services is exac- erbated by the increase of risk factors in patients such as immunodepression, chronic disease (diabetes, hypertension, heart disease, etc...), massive use of antibiotics due to multiple hospitalizations and repeat urinary tract infections (Figure 4).

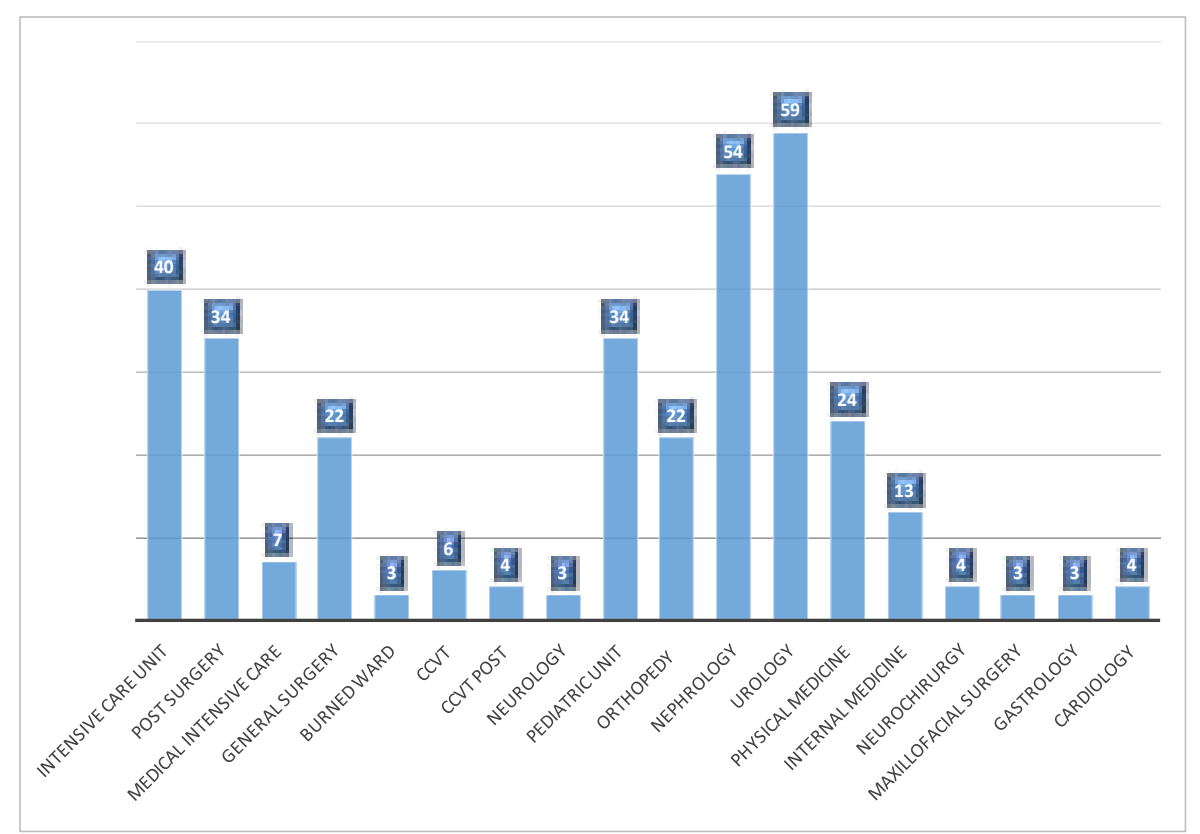

Figure 4: Distribution of ESBL producing strains between different wards in the university hospital Sahloul, Tunisia (2012-2014) 


\section{Mecanisms of resistance to cephalosporins}

Resistance to cephalosporins can be caused by production of ESBL and also of cephalosporinases. Enterobacteriaceae with AmpC $\beta$-lactamase are important causes of cephalosporin and cephamycin resistance 16. AmpC enzymes hydrolyze efficiently $3^{\text {rd }} \mathrm{GC}$ but it is not inhibited by clavulanic acid. In general class $C \beta$-lactamase constitutes a group of enzymes widely distributed in Enterobacteriaceae. The most clinically important acquired AmpC $\beta$-lactamases are CMY-2-like, ACC, and DHA types. The most prevalent mechanisms of resistance to several families of antibiotics especially cephalosporins among Gram-negative pathogens are represented by emergence of broad-spectrum $\beta$-lactamases. They have the property of being inhibited by clavulanic acid ${ }^{17}$.

ESBL producing organisms were first detected in Europe. First reports were from Germany and England ${ }^{18}$, and the vast majority of reports were from France ${ }^{19,20}$. In Tunisia, ESBL producing Enterobacteriaceae have been described as being the most important pathogens those causes nosocomial infections since their discovery at the end of the 80 years. The three major families are TEM, SHV and CTX-M, and are plasmid located.

In 1983, SHV-2 was isolated in Germany in Klebsiella ozaenae which possessed a $\beta$-lactamase which efficiently hydrolyzed cefotaxim and to a lesser ceftazidime ${ }^{21}$. In Tunisia, SHV-2 enzyme was the first ESBL described in 1984 in pediatric ward at the Charles Nicolle Hospital in K.pneumoniae strains ${ }^{19}$. Between 1984 and 1988, TEM enzymes were reported. Since, the frequency of $3^{\text {rd }}$ GC-resistant Enterobacteriaceae has increased significantly ${ }^{22}$. This enzyme is able to hydrolyze ampicillin at a greater rate than carbenicillin, oxacillin, or cephalotin, and has negligible activity against extended spectrum cephalosporins; it is inhibited by clavulanic acid. This enzyme largely dissimulated and replaced, the situation may have now turned out into a dominant CTX-M which hername reflects the potent hydrolytic activity against cefotaxim ${ }^{13}$, especially CTX-M-15 which emerged in an epidemic way. This enzyme has been described in the first time at the Charles Nicolle Hospital in Tunis in 2006. Since, it has been described several times in different hospitals and in different regions of the country ${ }^{23,24}$, for example, in Mahdia which a city located near to Sousse, CTX-M- ${ }^{15}$ was the ESBL the most isolated 15. In our hospital CTX-M-15 has been described in relation to NDM-1-producing multidrug-resistant strains ${ }^{24}$.

\section{b- Resistance to carbapenem}

Treatment options for bacterial infections have gradually reduced in front of increasing prevalence of antibiotic resistance and the lack of new antibiotic drug development ${ }^{3,25}$. Carbapenems such as imipenem and ertapenem are antibiotics used as first line for the treatment of severe infections caused by multi-resistant Enterobacteriaceae, such as Klebsiella pneumonia ${ }^{26}$. Carbapenemases are enzymes which are capable of breaking down most $\beta$-lactams including carbapenems, and thus conferring resistance to these drugs. Carbapenemase resistant Enterobacteriaceae have increasingly been reported worldwide in an alarming way in particular in Klebsiella pneumoniae ${ }^{27,28}$.

In our hospital, resistance to carbapenem molecules is mostly described in Klebsiella pneumoniae isolates. By introducing laboratory's data of resistance to ertapenem for the years 2010 and 2011, we have found that resistance to these molecules started from 2011 and has increased remarkably from 2012. The distribution of resistant strains between the hospital and the community shows that they are more isolated in the hospital (258/1250 and 84/910 respectively), but their presence in the community poses a real problem because we can no longer limit their emergence. It is difficult to modulate factors that enhance spread of carbapenemase producers in the community, because, these factors are multiple and associated with lack of hygiene, overuse of antibacterial drugs and also increased worldwide travel ${ }^{28}$.

The rate of resistance to ertapenem has increased from $2 \%$ in 2010 and 2011 to $17.7 \%$ in 2012. This rate decreased slightly in 2013 of $22 \%$ ( $0.21 \%$ in 2013 vs $0.26 \%$ in 2012) (Table*). This decrease is due to the rigorous application of hygiene measures especially in the intensive care units following training sessions for medical and paramedical personnel in order to limit their emergence. In 2014, the rate re-increases of 47\% (0.33\% in 2014 vs $0.21 \%$ in 2013 ) (Table 6). Concerning imipenem, resistance to this molecule increased from $0.38 \%$ in 2010 to $1.3 \%$ in 2014 . On the other hand, number of strains showing an intermediate sensitivity to imipenem has increased (11.6\% in 2014) (Figure 5). 
Table 6: Percentage of resistance in klebsiella pneumoniae compared to the total number of admission to the hospital in the period of three years

\begin{tabular}{|l|l|l|l|}
\hline Years & $\begin{array}{l}\text { Total of admission in hospital } \\
\text { wards }\end{array}$ & $\begin{array}{l}\text { Klebsiella pneumoniae } \\
\text { resistant to ertapenem }\end{array}$ & of resistance \\
\hline 2012 & 31506 & 83 & $0.26 \%$ \\
\hline 2013 & 29629 & 64 & $0.21 \%$ \\
\hline 2014 & 28428 & 94 & $0.33 \%$ \\
\hline
\end{tabular}

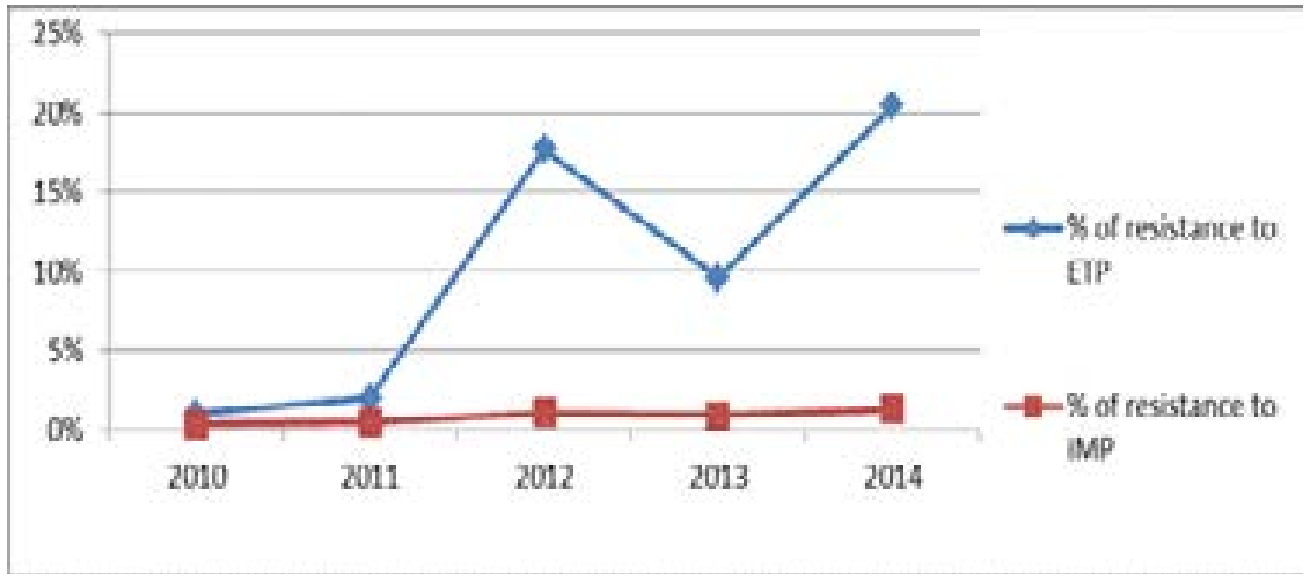

Figure 5: Evolution of resistance to imipenem and ertapenem from 2010 to 2014

Such as the results of ESBL producing strains, bacteria with carbapenem resistance mechanism are mostly isolated from samples coming from patient hospitalized in urology and intensive care unit departments, where there is a high flow of admission of patients who already have a favorable ground for the acquisition of multidrug-resistant bacterial infections. The rate of resistant strains has increased in an alarming way in the nephrology department in 2014.

\section{-Phenotypic analysis of carbapenem resistance mechanism}

The results of the Rosco discs realized on 241 Klebsiella pneumoniae resistant to ertapenem, which have been recovered from the laboratory preservation sack, allowed classifying strains into two major groups (Figure 6): i- Klebsiella pneumoniae strains possessing an enzymatic mechanism of carbapenem resistance by carbapenemases production. This group is divided into two subgroups: i-1- Metallo- $\beta$-lactamase producing strains: 17 strains showed increase in the diameter of inhibition of meropenem+dipicolinic acid disk compared with the disk of meropenem only. Those strains were characterized; they are NDM-1 producing strains ${ }^{24}$.

i-2- Oxacillinase-producing strains (OXA-48 like): 203 strains showed resistance to temocillin indicating the presence of class D carbapenemases.

In our hospital, class A carbapenemases especially KPC enzyme is not yet isolated.

ii- Strains possessing a non-enzymatic mechanism of resistance to carbapenems: 21 strains showed susceptibility to temocillin, a negative boronic acid and dipicolinic assays. 
In Sahloul hospital, carbapenem resistant Klebsiella pneumoniae was first described in 2008 in relation with overexpressing of AmpC cephalosporinase CMY-4 combined with loss of an outer membrane protein ${ }^{29}$. Since, no other cases have been described.

Our phenotypic study showed the dominance of temocillin-resistant Klebsiella pneumoniae, which is in favor of production of class D carbapenemase (OXA-48 like). OXA-48 has the property to hydrolyze carbapenems but not third generation cephalosporins; its activity is not inhibited by clavulanic acid. It is often associated with other $\beta$-lactamases, particularly ESBL, which contributes to multiresistance of strains $^{30}$.

In Tunisia, the first report of an OXA-48 producer was in $2010^{5}$, since then, numerous cases have been described her emergence in an endemic way. The derivative OXA204 has also been described ${ }^{31,32}$. Eight variants of OXA48 have been reported from different countries differing by few amino acid substitutions or deletions ${ }^{33,34}$ : OXA162 35, OXA-18136, OXA-163, OXA- OXA-232 34, OXA-244, OXA-245 37, OXA-247 38 and OXA-405 39. OXA-48 is mainly present around Mediterranean countries; its worldwide distribution includes countries of Europe especially France, Germany and Spain and countries of North Africa such as Tunisia and Morocco. The United States and Canada have been spared from the production of this enzyme.

Other than class D carbapenemases, metallo- $\beta$-lactamases have been also described in Tunisia. The first publication of an MBL in an Enterobacteriaceae specie dates back to 2005 with a VIM-4-producing Klebsiella pneumoniae in $\mathrm{Sfax}^{40}$. In 2012, NDM-1 has been described for the first time in Tunisia ${ }^{41}$. In our hospital NDM-1 was the subject of an emerging epidemic especially in intensive care unit $^{24}$.

In the world, the most isolated carbapenemase is KPC; this plasmid resistance is increasingly described. Recently it has been detected in Tunisia but not yet in our hospi$\operatorname{tal}^{42,43}$

\section{Evolution of resistance to colistin}

Polymyxin antibiotics, including colistin, are one of the few antibiotics still active on carbapenemase-producing Enterobacteriaceae. They are currently considered as last resort antibiotics drugs for treating infections due to multidrug resistant Gram-negative pathogens. This is an anti- biotic which cause cell membrane leakage. Unfortunately, resistance to this molecule has also been reported and still emerging worldwide. Mechanism of resistance to colistin in Enterobacteriaceae can be due to modification of lipid A with 4-amino-4-deoxy-L-arabinose and phosphoethanolamine which neutralizes the negative charge, or to acquisition of plasmid mediating gene named mcr-1, which encoded a phosphoethanolamine transferase that modifies the lipopolysaccharide structure of Gram-negative bacteria ${ }^{44}$. Colistin plasmid resistance (mcr-1 gene) in Enterobacteriaceae was first detected in China at the end of 2015. Its plasmid character allows it to be very easily transferable between bacteria ${ }^{44}$.

In our hospital, the introduction of colistin in the treatment of severe infections due to carbapenem-resistant strains has led to the appearance among isolated Klebsiella pneumoniae of resistance to colistin. Its frequency remains for the moment very limited. Nine strains only showed resistance to colistin during the three years of study, seven were observed in the urine samples; only one was isolated in a blood culture and one from a catheter.

In different countries of the world, more and more publications have spoken on the emergence of colistin resistant Klebsiella pneumoniae strains. In Taiwan, 17\% of carbapenem resistant Klebsiella pneumoniae are resistant to colistin $^{45}$. A surveillance study in Italy also revealed that $43 \%$ of carbapenemase-producing $K$. pneumoniae isolates were resistant to colistin ${ }^{46}$.

The new emerging resistances lead to a real therapeutic impasse. The situation is really alarming and requires taking and application of precautionary measures in order to limit the emergence of these multidrug resistant bacteria.

\section{Conclusion}

Our work showed the different profiles of antibiotic resistance in Klebsiella pneumoniae isolated at Sahloul hospital. The various mechanisms responsible for carbapenem resistance have also been demonstrated. The emergence of new resistances can lead to a therapeutic impasse with serious consequences on the health of infected patients or even the increase in mortality rates. It is therefore important to insist on compliance with hospital hygiene rules and the rational use of carbapenem to limit the spread of such enzymes. The development of other antibiotics molecules is therefore imperative to deal with infections caused by multiresistant bacteria. 


\section{Conflict of interest}

None declared.

\section{References}

1. Karah N, Sundsfjord A, Towner K and Samuelsen O. 2012. Insights into the global molecular epidemiology of carbapenem non-susceptible clones of Acinetobacter baumannii. Drug Resist Updat. 15(4):237-247. http://dx. doi.org/10.1016/j.drup.2012.06.001

2. Jesudason MV, Kandathil AJ, Balaji V. 2005. Comparison of two methods to detect carbapenemase \& metallo beta-lactamase production in clinical isolates. Indian J Med Res. 121(6):780-3. https://www.ncbi.nlm.nih.gov/ pubmed/16037624

3. Steinmann J, Kaase M, Gatermann S et al. 2011. Outbreak due to a Klebsiella pneumoniae strains harbouring KPC-2 and VIM-1 in a German university July 2010 to January 2011. Eurosurveillance. 16(33).pii: 19944. http://www.eurosurveillance.org/ViewArticle.aspx?ArticleId=19944

4. Souli M, Galani I, Antoniadou A et al. 2010. An outbreak of infection due to beta-lactamase Klebsiella pneumoniae carbapenemase 2 producing K.pneumoniae in a Greek university hospital : molecular characterization, epidemiology and outcomes. Clin infect Dis; 50(3):36473.https://academic.oup.com/cid/article-lookup/ doi/10.1086/649865

5. Ktari S, Arlet G, Mnif B et al. 2006. Emergence of multidrug resistant Klebsiella pneumoniae isolates producing VIM-4 metallo- $\beta$-lactamase, CTX-M extended spectrum $\beta$-lactamase and CMY-4 AmpC $\beta$-lactamase in a Tunisian university hospital. Antimicrob Agents Chemother. 50(12): 4198-4201. https://dx.doi.org/10.1128\%2FAAC.00663-06

6. Wang JT, Wu UI, Lauderdale TL et al. 2015. Carbapenem Non susceptible Enterobacteriaceae in Taiwan. PLoS One. 10(3): e0121668. https://doi.org/10.1371/journal. pone. 0121668

7. Lee Hyo-Jin, Jae-Ki Choi et al. 2016. Carbapenem resistant Enterobacteriacea : Prevalence and risk factors in a single community based hospital in Korea. Infect Chemother. 48(3):166-173. https://doi.org/10.3947/ic.2016.48.3.166 8. Podschun R, Ullmann U. 1998. Klebsiella spp. As nosocomial pathogens: epidemiology, taxonomy, typing methods and pathogenicity factors. Clin Microbiol Rev. 11(4): 589-603. https://www.ncbi.nlm.nih.gov/pmc/articles/ PMC88898/
9. Highsmith.K, Jarvis WR. 1985. Klebsiella pneumoniae: Selected virulence factors that contribute to pathogenicity. Infection Control. 6(2):75-7. https://www.ncbi.nlm. nih.gov/pubmed/2857687

10. Bouassida K, Jaidane M, Bouallegue O et al. 2016. Nosocomial urinary tract infections caused by extendd spectrum beta-lactamase uropathogens: Prevalence, pathogens, risk factors and strategies for infection control. Can Urol Assoc J. 10(3-4):E87-93. https://doi. org/10.5489/cuaj.3223

11. Carle Sylvie. 2009. La résistance aux antibiotiques: un enjeu de santé publique important. Pharmactuel.42:6-21. http://www.pharmactuel.com/pharmactuel/index.php/ pharmactuel/article/view/977

12. Christensen SC et Korner B. 1972. An endemic caused by multiresistant Klebsiella in an urological unit. Scand J Urol Nephrol. 6(3):232-8. https://www.ncbi.nlm. nih.gov/pubmed/4565200

13. Paterson DL et Bonomo RA. 2005. Extended spectrum beta-lactamases : a clinical update. Clinical Microbiology Reviews. 18(4):657-686. https://doi.org/10.1128/ CMR.18.4.657-686.2005

14. Gootz TD .1990. Discovery and development of new antimicrobial agents. Clin. Microbiol. Rev. 3(1): 13-31. https://www.ncbi.nlm.nih.gov/pmc/articles/ PMC358138/

15. Mansour W, Grami R, Ben Haj Khalifa A et al. 2015. Dissemination of multidrug-resistant bla CTX-M-15/ IncFIIk plasmids in Klebsiella pneumoniae isolates from hospital and community acquired human infections in Tunisia. Diagnostic Microbiol Infect Dis. 83(3): 298-304. https://doi.org/10.1016/j.diagmicrobio.2015.07.023

16. Jacoby GA.2009. AmpC B-lactamase. Clin Microbiol Rev. 22(1):161-182. https://doi.org/10.1128/ CMR.00036-08

17. Sibhghatulla S, Jamale F, Shazi S et al. 2015. Antibiotic resistance and extended spectrum beta-lactamases: Types, epidemiology and treatment. Saudi J Biol Sci. 22(1):90-101. https://dx.doi.org/10.1016\%2Fj.sjbs.2014.08.002

18. Dubois SK, Marriott MS and Amyes SG. 1995. TEM and SHV derived extended spectrum beta-lactamases: relationship between selection structure and function. $J$ Antimicrob Chemother. 35(1):7-22. https://www.ncbi.nlm. nih.gov/pubmed/7768784

19. Philippon A, Labia R, et Jacoby G. 1989. Extended spectrum beta-lactamases. Antimicrob Agents Chemother. 33(8):1131-1136. https://www.ncbi.nlm.nih.gov/ pubmed/2679367 
20. Sirot D, Recule C, Chaibi B et al. 1997. A complex mutant of TEM-1 beta-lactamase with mutations encountered in both IRT-4 and extended spectrum TEM15, produced by an Escherichia coli clinical isolate. $A n$ timicrob Agents Chemother. 41(6):322-1325. https://www. ncbi.nlm.nih.gov/pmc/articles/PMC163908/

21. Liakopoulos A, Mevius D et Ceccarelli D. 2016. A review of SHV extended spectrum beta-lactamases: $\mathrm{Ne}$ glected yet ubiquitous. Frontiers in Microbiology. 5;7 :1374. https://doi.org/10.3389/fmicb.2016.01374

22. Ben Hassen A, Fournier G, Kechrid A et al. 1990. Enzymatic resistance to cefotaxime in 56 strains of Klebsiella spp, Escherichia coli and Salmonella spp at a Tunisian hospital (1984-1988). Path Biol (Paris). 38(5):464-469. https://www.ncbi.nlm.nih.gov/pubmed/2195457

23. BaraniakA, fiett J, Hryniewiez W et al. 2002. Ceftazidime hydrolysingCTX-M-15 extended spectrum beta-lactamase (ESBL) in Poland. Journal Antimicrob chemother. 50(3): 393-396. https://www.ncbi.nlm.nih.gov/ pubmed/12205064

24. Messaoudi.A, Haenni M, Mansour W et al. 2016. ST147 NDM-1 producing Klebsiella pneumoniae spread in two Tunisian hospitals. I Antimicrob Chemother. 72(1):315-316. http://dx.doi.org/10.1093/jac/dkw401 25. Hyo Jin Lee, Jae Ki Choi, Sung Yeon Cho et al. 2016. Carbapenem resistant Enterobacteriacea : Prevalence and risk factors in a single community based hospital in Korea. Infect Chemother. 48(3):166-173.https://doi.org/10.3947/ ic.2016.48.3.166

26. Nordmann P, Cuzon G, Naas T.2009. The real threat of Klebsiella pneumoniae carbapenemase producing bacteria. Lancet Infect Dis. 9(4):228-36. https://doi. org/10.1016/S1473-3099(09)70054-4

27. Lee Chang-Ro, Jung Hun Lee, Kwang Seung Park, et al. 2016. Global dissemination of Carbapenemase producing Klebsiella pneumoniae: Epidemiology, genetic context, treatment options and detection methods. Front in Microbiol. 7:895. https://dx.doi.org/10.3389\%2Ffmicb.2016.00895

28. Nordmaan P, Naas T et Poirel L. 2011. Global spread of carbapenemase producing Enterobacteriaceae. Emerging Infectious Diseases. 17(10):1791-98. https://dx.doi. org/10.3201\%2Feid1710.110655

29. Dahmen S, Mansour W, Charfi K, et al. 2012. Imipenem resistance in Klebsiella pneumoniae is associated to the combination of plasmid-mediated CMY-4 AmpC $\beta$-lactamase and loss of an outer membrane protein. $M i$ - crob Drug Resist. 18(5):479-83. https://doi.org/10.1089/ mdr.2011.0214

30. Gülmez D, Woodford N, Palepou MF, et al.2008. Carbapenem-resistant Escherichia coli and Klebsiella pneumoniae isolates from Turkey with OXA-48-like carbapenemases and outer membrane protein loss. Int J $A n$ timicrob Agents. 31(6):523-6. https://doi.org/10.1016/j. ijantimicag.2008.01.017

31. Grami Raoudha, Wejdene Mansour, Anis Ben Haj Khalifa et al. 2016. Emergence of ST147 Klebsiella pneumonia producing OXA-204 carbapenemase in a university Hospital Tunisia. Microbial Drug Resistance. 22(2):137140. https://doi.org/10.1089/mdr.2014.0278

32. Charfi K, Mansour W, Ben Haj Khalifa A et al. 2015. Emergence of OXA-204 $\beta$-lactamase in Tunisia. Diagnostic Microbioligy and Infection Disease. 82(4):314-317. https:/ / doi.org/10.1016/j.diagmicrobio.2015.04.003

33. Potron A, Poirel L, Rondinaud E, P Nordmann. 2013(a). Intercontinental spread of OXA-48 beta-lactamase producing Enterobacteriacae over a 11 year period. 2001 to 2011. Euro Surveill. 18(31).pii:20549. http:/ /www. eurosurveillance.org/ViewArticle.aspx?ArticleId=20549 34. Potron A, PondinaudE, Poirel L et al.2013(b). Genetic and biochemical characterization of OXA-232 a carbapenem-hydrolysing class D B-lactamase from Enterobacteriacae. Int J Antimicrob Agents. 41(4):325-9. https:// doi.org/10.1016/j.ijantimicag.2012.11.007

35. Kasap M, Torol S, Kolayli F, Dudar D et al. 2013. OXA-162 a novel variant of OXA-48 displays extended hydrolytic activity towards imipenem, meropenem and doripenem. J Enzyme Inbib Med Chem. 28(5):990-6. https://doi.org/10.3109/14756366.2012.702343

36. Poirel L, Potron A and Nordmann P. 2012. OXA48 like carbapenemase: the phantom menace. J Antimicrob Chemother. 67(7):1597-606. https://doi.org/10.1093/jac/ dks121

37. Oteo J, Hernandez JM, Espasa M et al. 2013.Emergence of OXA-48 producing Klebsiella pneumonia and the novel carbapenemases OXA-244 and OXA-245 in Spain. Journal of Antimicrobial Chemotherapy. 68 (2):317-21. https://doi.org/10.1093/jac/dks383

38. Gomez S, Pasteran F, Faccone D et al. 2013. Intrapatient emergence of OXA-247: A novel carbapenemase found in a patient previously infected with OXA-163 producing Klebsiella pneumonia. Clinical Microbiology and Infection. 19(5):E233-5. https://doi.org/10.1111/14690691.12142 
39. Voulgari E, Poulou A, Dimitroulia E, Polti L et al. 2015. Emergence of OXA-162 carbapenemase and DHA-1 AmpC cephalosporinases producing ST 11 Klebsiella pneumonia causing community-onset infection in Greece. Antimicrobial Agents and chemotherapy. 60(3):1862-4. https://doi.org/10.1128/AAC.01514-15

40. Ktari S, Arlet G, Mnif B et al. 2006. Emergence of multidrug resistance Klebsiella pneumoniae isolates producing VIM-4 metallo-beta-lactamase, CTX-M-15 extended spectrum beta-lactamase and CMY-4 AmpC beta-lactamase in a Tunisian university hospital. Antimicrob Agents Chemother. 50(12):4198-4201. https://doi. org/10.1128/AAC.00663-06

41. Ben Nasr A, Decret D, Compain F et al. 2013. Emergence of NDM-1 in association with OXA-48 in Klebsiella pneumoniae from Tunisia. Antimicrob Agents Chemother. 57(8): 4089-90. https://dx.doi.org/10.1128\%2FAAC.00536-13

42. Ben Tanfous F, Andrea Alonso C, Achour W et al. 2017. First description of KPC-2 producing Escherichia coli and ST15 OXA-48 positive Klebsiella pneumoniae in Tunisia. Microbial Drug Resistance. 2" (3): 365-375. https:/ / doi.org/10.1089/mdr.2016.0090
43. Battikh H, Harchay C, Dekhili A et al. 2016. Clonal spread of colistin resistant Klebsiella pneumoniae coproducing KPC and VIM carbapenemases in neonates at a Tunisian university hospital. Microbial Drug Resistance. 23(4):468-472. https://doi.org/10.1089/mdr.2016.0175 44. Dobias Jan, Poirel Laurent, Nordmann Patrice. 2017. Cross resistance to human cationic antimicrobial peptides and to polymyxins mediated by the plasmid encoded mcr-1. Clinical Microbiology and Infection. Pii: S1198-743X(17):30184-2.https://doi.org/10.1016/j. cmi.2017.03.015

45. Chiu SK, Wu TL, Chuang YCet al. 2013. National surveillance study on carbapenem non-susceptible Klebsiella pneumoniae in Taiwan: the emergence and rapid dissemination of KPC-2 carbapenemase. PLoS One. 8(7):e69428. https://doi.org/10.1371/journal.pone.0069428

46. Monaco M, Giani T, Raffone M et al. 2014. Colistin resistance superimposed to endemic carbapenem-resistant Klebsiella pneumoniae: a rapidly evolving problem in Italy, November 2013 to April 2014. Euro Surveill. 19(42):pii20939. http://dx.doi.org/10.2807/1560-7917. ES2014.19.42.20939 